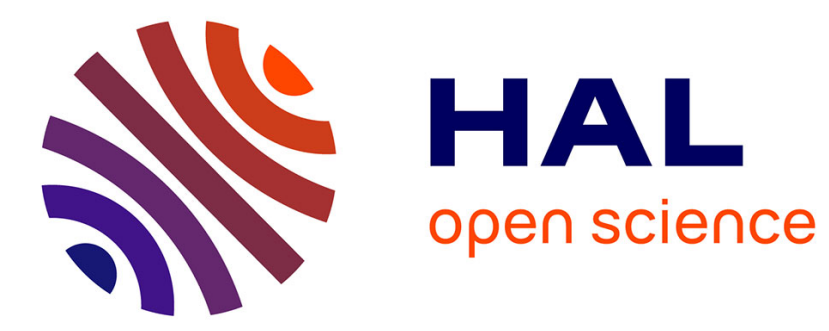

\title{
Artistes versus musées, enjeux d'une pensée critique Elvan Zabunyan
}

\section{To cite this version:}

Elvan Zabunyan. Artistes versus musées, enjeux d'une pensée critique. Cahiers philosophiques, 2011, 124 (1), pp.66 -83. 10.3917/caph.124.0066 . hal-01626306

\section{HAL Id: hal-01626306 \\ https://hal.univ-rennes2.fr/hal-01626306}

Submitted on 30 Oct 2017

HAL is a multi-disciplinary open access archive for the deposit and dissemination of scientific research documents, whether they are published or not. The documents may come from teaching and research institutions in France or abroad, or from public or private research centers.
L'archive ouverte pluridisciplinaire HAL, est destinée au dépôt et à la diffusion de documents scientifiques de niveau recherche, publiés ou non, émanant des établissements d'enseignement et de recherche français ou étrangers, des laboratoires publics ou privés. 


\title{
ARTISTES VERSUS MUSÉES, ENJEUX D'UNE PENSÉE CRITIQUE Elvan Zabunyan
}

\author{
Réseau Canopé | « Cahiers philosophiques »
}

$2011 / 1 n^{\circ} 124 \mid$ pages 66 à 83

ISSN 0241-2799

Article disponible en ligne à l'adresse :

https://www.cairn.info/revue-cahiers-philosophiques1-2011-1-page-66.htm

\section{Pour citer cet article :}

Elvan Zabunyan, «Artistes versus musées, enjeux d'une pensée critique », Cahiers philosophiques 2011/1 ( $\left.\mathrm{n}^{\circ} 124\right)$, p. 66-83.

DOI 10.3917/caph.124.0066

Distribution électronique Cairn.info pour Réseau Canopé.

(c) Réseau Canopé. Tous droits réservés pour tous pays.

La reproduction ou représentation de cet article, notamment par photocopie, n'est autorisée que dans les limites des conditions générales d'utilisation du site ou, le cas échéant, des conditions générales de la licence souscrite par votre établissement. Toute autre reproduction ou représentation, en tout ou partie, sous quelque forme et de quelque manière que ce soit, est interdite sauf accord préalable et écrit de l'éditeur, en dehors des cas prévus par la législation en vigueur en France. Il est précisé que son stockage dans une base de données est également interdit. 


\title{
Le musée
}

\section{ARTISTES VERSUS MUSÉES, ENJEUX D'UNE PENSÉE CRITIQUE}

Elvan Zabunyan

\begin{abstract}
À la fin des années 1960, de nouvelles formes d'expression artistique émergent dans un contexte troublé où revendications politiques, sociales, économiques et culturelles contribuent à transformer radicalement les rapports entre artistes et institutions muséales. Alors que ces dernières, fortement attaquées pour leur conservatisme et leur posture autoritaire, tentent de trouver un compromis avec leurs détracteurs, les artistes eux s'engagent dans des processus créatifs qui contournent l'hégémonie tant culturelle qu'économique du musée en s'en emparant comme d'un outil expérimental. Marcel Broodthaers et Fred Wilson, chacun à leur manière, analysent avec acuité le rôle idéologique du musée dans leurs expositions.
\end{abstract}

es musées sont les sépultures familiales des œuvres d'art» dit sans détour la citation de Theodor Adorno que l'historien d'art américain Douglas Crimp choisit de placer en exergue de son texte On the Museum's Ruins en 1980. Depuis sa naissance au XIX ${ }^{\mathrm{e}}$ siècle, le musée d'Art contemporain, exposant, archivant, conservant la mémoire des beaux-arts, a été le terrain de bataille de pratiques artistiques et de prises de position théoriques contestant l'affirmation universaliste de la modernité institutionnalisée. Les artistes constructivistes, dada et surréalistes, définissant les paradigmes des avant-gardes historiques dans les années 1920, sont parmi les premiers à destituer l'hégémonie du musée en faisant tomber de leur piédestal - au sens propre et au sens figuré - les œuvres contemporaines. Par opposition aux formes d'expression traditionnelles telles la peinture ou la sculpture, les nouvelles approches artistiques se détachent du courant institué par l'histoire de l'art et par l'académie. 
Elles construisent leur propre système de valeurs, principalement fondé sur une liberté de création : tout matériau peut être assimilé à un matériau artistique, tout objet peut endosser le rôle d'objet d'art, toute image (reproduite ou reproductible) peut devenir référence visuelle, toute action - qu'elle s'inscrive dans un espace-temps réduit ou en expansion - peut être considérée, malgré son caractère éphémère, comme une forme d'expression artistique.

Cette liberté de l'artiste est celle d'un processus lui-même libéré des contraintes conventionnelles ; ni le créateur, ni son travail ne se conçoivent enfermés au sein d'un système régi par les musées, les galeries, les collectionneurs et les critiques d'art. L'artiste est au cœur d'un système qui vient confirmer le parallèle d'Adorno entre le musée et l'enterrement des œuvres. Son rôle va être de renverser les valeurs académiques et de démontrer par son travail que le contexte de production et la forme de la représentation qui en découle s'inscrivent dans une remise en cause radicale de l'autorité muséale.

Les œuvres d'art prisonnières de I'univers muséal le sont aussi de leur histoire
Le titre de l'essai de Crimp esquisse la possibilité d'une construction théorique dont la fondation est la ruine du musée. La méthode de l'archéologue à laquelle se réfère l'auteur s'appuie sur la pensée foucaldienne inaugurant une analyse poststructuraliste où la connaissance est organisée selon un modèle discursif tentant de contrarier le pouvoir institutionnel. «Foucault s'est concentré sur les institutions modernes de l'enfermement - l'asile, la clinique, la prison - et leur formation discursive respective - la folie, la maladie, la criminalité. Il y a une autre institution similaire en attente d'une analyse archéologique - le musée - et une autre discipline - l'histoire de l'art. Ils sont avec la photographie et peut-être, plus précisément, la répression et l'usage sélectif de la photographie, la condition liminaire du discours que l'on nomme art moderne ${ }^{1}$. » Les œuvres d'art prisonnières de l'univers muséal le sont aussi de leur histoire. Les artistes, les premiers, cherchent alors à intégrer à leur processus créatif des moyens de contredire l'élitisme qui se trouve rattaché aux fondements même de la modernité. Crimp fait partie de la génération d'historiens et théoriciens de l'art appartenant à la gauche marxiste américaine ayant lu avec attention les textes de philosophes qui redéfinissent la place de l'auteur. Walter Benjamin avec «L'auteur comme producteur » (1934, traduit en anglais en 1969), Roland Barthes avec « La mort de l'auteur » (1968), Michel Foucault avec «Qu'est-ce qu'un auteur ? (1969) sont des références incontournables pour la critique d'art de l'époque. Tous les trois s'appuient sur des exemples littéraires pour articuler leurs propositions théoriques².

1. Douglas Crimp, "On the Museum's Ruins », October n 13, été 1980, p. 45.

2. Walter Benjamin, "L'auteur comme producteur ", in Essais sur Bertold Brecht, Paris, Maspero, 1978, p. 107-128; Roland Barthes, "La mort de l'auteur " in Le Bruissement de la langue, Essais critiques IV, Paris, Seuil, 1984, p. 61-67 ; Michel Foucault, "Qu'est-ce qu'un auteur ? » in Dits et Écrits I, 1954-1975, Paris, Gallimard, 2001, p. 817-849. 
Lors d'une conférence (prononcée le 27 avril 1934 à Paris), Benjamin, dans un commentaire sur le théâtre épique de Bertold Brecht, analyse les rapports sociaux à la lumière des rapports de production et rejoint l'« éloignement de l'Auteur » défini par Barthes. «Avec Brecht, on pourrait parler ici d'un véritable “distancement”, l'Auteur diminuant comme une figurine tout au bout de la scène littéraire n'est pas seulement un fait historique ou un acte d'écriture, il transforme de fond en comble le texte moderne (ou - ce qui est la même chose - le texte est désormais fait et lu de telle sorte qu'en lui à tous ses niveaux l'auteur s'absente) ${ }^{3}$. » C'est aussi un écrivain que convoque Foucault en adossant le texte de sa conférence (prononcée le 22 février 1969 au Collège de France) à cette phrase de Samuel Beckett : «Qu'importe qui parle, quelqu'un a dit qu'importe qui parle. » « Dans cette indifférence, écrit Foucault, je crois qu'il faut reconnaître un des principes éthiques fondamentaux de l'écriture contemporaine », avant de poursuivre un peu plus loin : «Si un individu n'était pas un auteur, est-ce qu'on pourrait dire que ce qu'il a écrit ou dit, ce qu'il a laissé dans ses papiers, ce qu'on a pu rapporter de ses propos pourrait être appelé une œuvre ${ }^{4}$ ? $\gg$ Cette réflexivité entre l'auteur et sa création est celle que les philosophes contredisent, de la même manière que les artistes eux-mêmes optent pour des démarches qui prônent une attitude critique et contestataire face à l'institution artistique et à son exploitation de l'art comme marchandise. En décidant de produire un art libéré des techniques académiques et de leurs contraintes, ils redéfinissent les notions d'espace, de temps, de forme et de représentation en les adaptant à la réalité culturelle, sociale et politique dans laquelle ils évoluent. L'inscription du travail artistique dans un espace intrinsèque à ce processus permet de redéfinir le contexte de sa diffusion et de sa réception et a pour conséquence une perspective sans précédent où l'artiste déclare ouverte la possibilité d'élever l'idée de l'art à un statut artistique qui peut exister sans « objet ». Le statut « objet» de l'œuvre ainsi dépassé mène à une réflexion reposant sur la «dématérialisation » du travail plastique au profit d'un concept. Au-delà d'une simple considération esthétique, utilisées comme outils méthodologiques, des pensées pluridisciplinaires - philosophique, psychanalytique, historique, sociologique, économique - donnent naissance à de nouvelles formes d'analyses de l'art.

La proximité des dates de publication des essais cités - et l'on pourrait ajouter à la liste la Théorie esthétique, ouvrage inachevé et posthume paru en 1970 qu'Adorno souhaitait dédier à Beckett - confirme une nécessité qui consiste à trouver des points de jonction entre événements politiques et production artistique. Car cet effacement de l'auteur en tant qu'individu isolé va de pair avec, dès le milieu des années 1960, l'engagement politique collectif partout dans le monde, et en particulier de façon très médiatisée dans les pays européens et en Amérique du Nord. Le mouvement des droits civiques américains, la guerre du Vietnam, les événe- 
ments de mai 1968, les répressions policières, l'invasion du Cambodge, le mouvement de libération des femmes, les libertés bafouées en Amérique du Sud provoquent des contestations au sein desquelles la communauté artistique s'engage avec radicalité.

Les activités militantes se développent dans un climat politique tendu qui voit de plus en plus fréquemment des coalitions artistiques s'organiser pour lutter contre les injustices propres au statut des artistes et tenter d'obtenir une reconnaissance de leurs créations. Ainsi, en 1969, est fondée l'Artworkers Coalition (AWC), qui regroupe des artistes de diverses origines au sein d'une organisation condamnant la discrimination raciale $\mathrm{du}$

Les activités

militantes se développent dans un climat politique tendu Museum of Modern Art et réclamant un nouveau programme de réforme de la politique générale de l'institution, avec notamment la gratuité d'accès pour tous. Au début de l'année 1970, l’AWC lance une importante action antimilitariste à la suite des bombardements américains au Cambodge et de la répression sanglante des mouvements étudiants à

l'université d'État de Kent, à Jackson et Augusta. Robert Morris, qui joue un rôle important dans la grève, retire ses œuvres du Pavillon américain à la Biennale de Venise en signe de protestation. La grève artistique (art strike) provoque la fermeture de nombreux musées et galeries de Manhattan et suscite une immense manifestation sur les marches du Metropolitan - seul à rester ouvert. « Grève de l'art contre le racisme, la guerre et la répression » proclament les manifestants. Les musées et leurs équipes comprennent qu'une politique basée sur une muséologie qui ne prendrait pas en compte la réalité de ces contestations fermerait encore plus les portes au dialogue. Le Museum of Modern Art, au centre des critiques et point géographique culminant des manifestations, répond à cette nouvelle situation en organisant en 1970 une exposition intitulée Information. Une centaine d'artistes européens et américains sont réunis par Kynaston McShine, alors jeune conservateur du département de peinture et de sculpture. Le catalogue de l'exposition est un forum ouvert où chaque artiste apporte sa contribution de façon libre. Le matériel présenté souligne une colère vive face aux crises sociales, politiques et économiques générales qui marquent l'année 1970. Dans son introduction, McShine indique bien l'impossibilité de rester indifférent aux événements contemporains en continuant un travail de conservation ou de création qui ne les prendraient nullement en compte. Il confirme le lien insécable entre les pratiques artistiques et la réception des faits politiques : "Si vous êtes un artiste au Brésil, vous connaissez au moins un ami en train d'être torturé ; si vous êtes un artiste en Argentine, vous avez certainement eu un voisin qui a été emprisonné pour avoir eu les cheveux longs ou pour ne pas avoir été "correctement" habillé ; et si vous habitez les États-Unis, vous pouvez craindre d'être tué, à l'université, dans votre lit ou, plus formellement, en Indochine. Il peut sembler trop déplacé, voire absurde, de se réveiller le matin, entrer dans une pièce et appliquer des touches de peinture sorties d'un petit tube sur le carré d'une toile. 
Qu'est-ce qu'un jeune artiste peut faire qui pourrait sembler pertinent et significatif 5 ? »

Alors que la position individuelle de Kynaston McShine indique clairement une ouverture politique et un regard attentif sur l'actualité, si le MoMA décide de lui confier le commissariat d'une telle exposition, il ne s'agit pas d'un simple mea culpa institutionnel. Il est aussi question ici de stratégie. Sans avoir pris ouvertement position contre la guerre du Vietnam, mais étant devenu le sujet et le lieu de la contestation et alors qu'il subit les attaques de l'AWC, le musée décide de réagir à chaud aux événements, conscient que les liens entre les questions politiques et artistiques ne sont pas juste des positions idéologiques appartement à un champ ou un autre mais que des liens méthodologiques intrinsèques sont en train de se former. La création contemporaine qui bouscule les formes d'art traditionnelles s'engage nécessairement dans de nouvelles conditions de (re)présentation que le musée, en tant qu'espace architectural accueillant et réalisant des expositions, va devoir prendre en compte pour confirmer son adhésion aux pratiques artistiques, quitte à produire un écartèlement entre sa position muséographique première et sa programmation en cours.

Le titre de l'exposition, Information, résonne comme un mot d'ordre en cette période de transition, notamment pour des pratiques qui, se détournant des matériaux habituels, créent des ponts vers la philosophie et la littérature en s'appropriant les codes et formes du langage. L'une des directions les plus radicales est celle de l'art conceptuel qui s'engage dans une reconfiguration du processus de création ${ }^{6}$. Comme l'indique l'artiste Robert Morris : «Ce que l'art désormais détient est un produit sujet à mutation qui n'a plus besoin de parvenir à une fin, dans le temps ou dans l'espace. L'idée que l'œuvre relève d'un processus irréversible, aboutissant à l'objet-icône statique, n'a plus beaucoup de pertinence aujourd'hui ${ }^{7}$. » La redéfinition de la notion de représentation artistique entraîne la problématisation des pratiques contemporaines de la décennie 1960 par le biais de paramètres propres à l'art lui-même. « À un niveau pratique, écrit Lucy Lippard, les artistes conceptuels offrirent une vision très claire de la fonction et de la place supposées de l'art ; poussant jusqu'au bout l'utopie, certains essayèrent de visualiser un nouveau monde reflété ou inspiré par l'art [...] Selon moi, l'art conceptuel est une œuvre dans laquelle l'idée

5. Kynaston L. McShine, "Essay ", dans le catalogue de l'exposition Information, New York, Museum of Modern Art, 1970, p. 138.

6. Le premier à avoir utilisé les termes de " concept art » est Henry Flynt en juin ou juillet 1961, dans son essai du même nom : "Le Concept Art est avant tout un art dont les matériaux sont des concepts, de la même manière que le matériau de la musique est le son. Dans la mesure où les concepts sont étroitement liés au langage, le Concept Art est une forme d'art dont le matériau est langage. " L'essai a été publié pour la première fois en 1963 dans l'ouvrage édité par l'artiste-performer La Monte Young, An Anthology (New York, Éditions La Monte Young, 1963).

7. Cette analyse de Robert Morris est parue dans la seconde partie du catalogue de l'exposition Conceptuel Art and Conceptual Aspects intitulée "Information 2 " et regroupant un certain nombre de citations et de formules spécifiques à une définition de l'art conceptuel. L'exposition s'est tenue entre le 10 avril et le 25 août 1970 au New York Cultural Center. Organisée par Donald Karshan, elle était la première manifestation d'art conceptuel s'inscrivant dans une institution muséale de cette envergure. Conceptuel Art and Conceptual Aspects, New York, The New York Cultural Center, 1970, p. 47. 
est primordiale et la forme matérielle, secondaire, légère, éphémère, bon marché, modeste ou "dématérialisée"8. » La déconstruction du système traditionnel de l'art se fait ainsi par le biais d'expérimentations quotidiennes qui peuvent rester à l'état d'expérimentations - et malgré tout exister comme travail artistique - mais aussi suivre un schéma plus conforme quand elles sont réalisées formellement. Ainsi Joseph Kosuth, dans son concept d'œuvres substituées à des « propositions analytiques », développe-t-il un art pensé comme son propre objet de définition, en s'appuyant notamment sur sa formule phare, « art as idea as idea».

Cet art défini par son concept devient également une information sur l'art et ses paramètres. Cette information, transmise par le biais de diverses publications - essais, manifestes, catalogues, etc. -, permet de nourrir, d'influencer, les pratiques d'autres artistes qui viendraient à en prendre connaissance. La diffusion des informations permet dès lors de dépasser les barrières géographiques, rendant possibles les rencontres et les communications entre les travaux artistiques à travers les régions et les pays. Cette forme de transmission devient elle-même un médium de travail pour les artistes qui intègrent l'idée de l'information à leur processus de création, rendant celui-ci proche d'une recherche sociologique. C'est notamment le cas d'un artiste comme Hans Haacke qui construit son œuvre sur une base de données révélant et dénonçant les différents systèmes de pouvoir, qu'ils soient économique, politique ou culturel, tout en critiquant leurs implications au sein de la réalité artistique institutionnelle. Lors d'un entretien en 1971, Haacke remarquait ainsi : «Une information présentée au bon moment et à la bonne place peut s'avérer très puissante. Elle peut affecter le tissu social dans son ensemble. Le principe du travail est de penser en termes de systèmes : la production de systèmes, les interférences avec les systèmes existants et leur dénonciation. Les systèmes peuvent être physiques, biologiques ou sociaux ${ }^{9}$. »

L'art conceptuel questionne directement les problématiques relatives à la place de l'auteur et remet en cause la définition habituelle de l'œuvre d'art. Dans ce contexte, les artistes réalisent souvent des travaux qui ne sont pas susceptibles d'être intégrés au marché de l'art. Même exposées dans des galeries ou des musées, leurs productions perdent leur statut premier d'objets d'échange marchand. Leur pouvoir initial perdu, galeries et musées réinventent dès lors de nouvelles formes de collaboration avec les artistes, renversant sur le modèle proposé par Benjamin l'équilibre entre les rapports de production et les rapports sociaux, permettant

8. En 1967, Lucy Lippard et John Chandler écrivent un article intitulé "The Dematerialization of Art », qui paraît en février 1968 dans la revue Art International et dans lequel ils observent l'émergence de ce qu'ils appellent l'« art ultraconceptuel » (par opposition à toutes les autres formes d'art - art minimal ou land art notamment -, qui apparaissent comme « anormalement cérébrales ») qui jaillit de deux sources complémentaires : I'art comme idée et l'art comme action. En 1973, Lucy Lippard publie sa remarquable compilation de tous les événements et micro-événements survenus au sein de l'art dit " conceptuel » entre 1966 et 1972 : Six Years: The Dematerialization of the Art Object from 1966 to $1972 \ldots$, New York, Praeger, 1973.

9. Jeanne Siegel, "An Interview with Hans Haacke », Arts Magazine vol. 45, n 7, mai 1971, p. 21. 
aux artistes de créer une œuvre où l'institution publique ou privée se retrouve au cœur de la critique. Par ce basculement de paradigmes, les réalisations des artistes s'ancrent dans le musée en y menant une exploration archéologique minutieuse où, étape après étape, la fonction muséale se retrouve elle-même au sein d'un processus conceptuel soulignant ses limites tout en nourrissant les recherches artistiques et théoriques. C'est au sein de ce paradoxe que conservateurs de musée et artistes tentent de trouver des terrains d'entente, les premiers étant conscients de l'importance historique et culturelle des transformations artistiques initiées par les seconds.

La notion d'auteur est convoquée de façon

Les réalisations des artistes s'ancrent dans le musée en y menant une exploration archéologique renouvelée et si sa disparition n'est pas annoncée, les fonctions propres aux rôles sociaux imposés se retrouvent déstabilisées. «On dit en effet (et c'est encore une thèse bien familière) que le propre de la critique, écrit Foucault, n'est pas de dégager les rapports de l'œuvre à l'auteur, ni de vouloir reconstituer à travers des textes une pensée ou une expérience, elle doit plutôt analyser l'œuvre dans sa structure, dans son architecture, dans sa forme intrinsèque et dans le jeu des relations internes. Or il faut aussitôt poser un problème : "qu'est-ce qu'une œuvre ? qu'est-ce donc que cette curieuse unité qu'on désigne du nom d'œuvre ? de quels éléments est-elle composée ? une œuvre, n'est-ce pas ce qu'a écrit celui qui est un auteur ?” On voit les difficultés surgir ${ }^{10}$. »

Vingt-neuf ans après Information, Kynaston McShine organise au MoMA une exposition qui tente de résumer les trois décennies pendant lesquelles querelles, rencontres, expériences marquent les liens que les artistes établissent avec les musées, prenant ces derniers, tour à tour comme objets d'étude, de critique, d'inspiration, de confrontation. The Museum as Muse, Artists Reflects est un titre évocateur où le renvoi au terme de «muse » résonne à la fois avec nostalgie et ironie. Du musée comme sépulture au musée comme muse, le chemin parcouru est grand. De lieu de conservation des œuvres, le musée devient le sujet même sur lequel travaillent les artistes. Au-delà du dialogue et du compromis, ce qui apparaît ici est que l'effort fourni par les deux parties indique à quel point ces changements sont nécessaires dans le contexte de l'art contemporain pour que les œuvres créées puissent exister au sein du musée, même sans être conformes à ses principes. Le bras de fer n'en est pas pour autant terminé mais, pour continuer à exister en tant que structure institutionnelle accueillant des artistes vivants, le musée se doit de céder sur certaines rigidités idéologiques en autorisant les artistes qui ont choisi d'en faire leur espace de travail, d'y créer. L'atelier n'est plus le lieu unique où l'art est réalisé, il reste un espace expérimental ; l'œuvre finie existe, elle, 
directement dans l'espace d'exposition. On parle alors d'art dit « d'installation » et d'art « in situ» réalisé par des artistes «post-ateliers ». La volonté de changer le sens des accrochages d'exposition traditionnels implique une relation nouvelle au support destiné à accueillir les œuvres. Le mur jusquelà privilégié cède par exemple sa place au sol. Non seulement les formes traditionnelles de représentations ne suffisent plus à exprimer les concepts artistiques engagés de l'époque mais en outre, en sortant des cadres (au sens propre autant que figuré) picturaux et sculpturaux, les artistes rendent par là même impossible le déplacement de l'œuvre créée. Ils refusent d'en faire un produit qui intégrerait les voies habituelles de l'échange commercial et inscrivent spécifiquement leur œuvre dans l'espace architectural. Mais changer les paramètres d'exposition revient aussi pour le musée à revoir les paramètres de sécurité, de circulation, d'accueil du public, qui restent eux partie intégrante du cahier des charges de l'institution. Si l'artiste acquiert une grande liberté dans l'agencement de son travail - il a par exemple la possibilité de garder la maîtrise de son œuvre en incluant notamment des clauses de montage ou de présentation lors de toute exposition -, il est obligé aussi de s'adapter aux limites du musée, même s'il cherche à les repousser. Les artistes choisissent le musée comme sujet car ils en ont une connaissance réelle. C'est par le musée qu'ils sont formés, c'est en visitant une exposition qu'ils se familiarisent avec les œuvres, c'est en étudiant les collections qu'ils évaluent les équilibres entre les acquisitions et le cours de l'histoire de l'art. C'est ce que souligne McShine dans son introduction : «Ce qui est fascinant dans la relation entre les artistes et les musées est que les artistes ont étudié tous les aspects du musée comme s'ils disséquaient un organisme. De même, la façon dont ils intègrent le musée dans leur travail va au-delà d'une simple considération pragmatique, leur intérêt est bien en partie professionnel : leur sens de ce que le musée signifie en termes d'acceptation publique rend un certain nombre d'entre eux impatients d'être représentés par des collections muséales et inquiets s'ils en sont absents. D'autres, pendant ce temps, se demandent si oui ou non leur travail devrait être présenté dans un musée, puisqu'y être est une manière de céder à l'establishment. Dans chaque cas, les artistes sont le plus souvent en train de lutter avec leur dépendance du musée pour garantir leur place dans l'histoire de l'art ${ }^{11}$. »

Dans les années 1920 et 1930, alors qu'il est installé aux États-Unis, Marcel Duchamp est l'un des premiers à inaugurer des relations différentes entre les artistes et les musées en étant notamment conseiller de plusieurs collectionneurs et d'institutions ; il permet dès lors d'orienter les achats vers des valeurs inédites - ses propres œuvres, dont les ready-mades, en faisant partie.

Alors que les catégories artistiques habituelles glissent vers des significations qui ne répondent plus aux critères reconnus, le musée s'interroge sur la façon de faire évoluer méthodes de travail et relations

11. Kynaston McShine, "Introduction " in The Museum as Muse, Artists Reflects, catalogue d'exposition, New York, Museum of Modern Art, 1999, p. 11. 
professionnelles lorsque les artistes provoquent des ruptures irréversibles. Dans la continuité de ce que Duchamp instaure, les juxtapositions entre art et idée, art et matériau, art et représentation, sont poussées jusqu'à leur limite dans les années 1960. Les principes de l'art conceptuel ouvrent vers une large réflexion esthétique. Les praticiens, à cette époque, non seulement se rapprochent de la théorie, mais construisent en outre leur travail visuel comme des penseurs ou des intellectuels. L'évolution du vocabulaire indique que l'on ne parle plus d' « œuvre » mais de «travail»; de même, l'art visuel rejoint l'art verbal. Ce sont aussi les migrations sémantiques qui caractérisent la transformation même de la réception : on regarde les mots, on lit les images, les poèmes sont des « événements », les arts plastiques ou visuels sont « joués » («performed»). Dans le contexte muséal, le renversement s'effectue à partir du moment où l'artiste s'approprie le concept d'archive ou de collection pour en faire l'outil de sa propre pratique. En appliquant les principes muséologiques à la production de l'art, certains artistes soulèvent des questions qui reflètent, plus que les approches théoriques, la réalité critique des processus mis en œuvre.

Un autre déplacement est perceptible : alors que certains artistes décident de faire du musée leur atelier, d'autres utilisent leur espace de travail pour le déclarer « musée ». Les guillemets renvoient ici volontairement à la fiction qu'une telle idée défend. Car un musée dans un atelier ne peut être que fictif, surtout lorsqu'il est ambulant. C'est ce que réalise Marcel Broodthaers, artiste belge (né à Bruxelles en 1924, mort à Cologne en 1976), lorsqu'il crée en 1968 et pour quatre ans son Musée d'art moderne, Département des Aigles ${ }^{12}$. En 1964, la déclaration de l'artiste, écrivain et poète devient fameuse : «Moi aussi je me suis demandé si je ne pouvais pas vendre quelque chose et réussir dans la vie, cela fait un moment déjà que je ne suis bon à rien. Je suis âgé de quarante ans... L'idée d'inventer quelque chose d'insincère me traversa l'esprit et je me mis aussitôt au travail, au bout de trois mois, je montrai ma production à Édouard Toussaint, le propriétaire de la galerie Saint-Laurent. Mais c'est de l'art dit-il, et j'exposerais volontiers tout ça. D'accord lui répondis-je. Si je vends quelque chose, il prendra $30 \%$, ce sont parait-il des conditions normales, certaines galeries prenant $75 \%$. Ce que c'est? En fait des objets ${ }^{13}$. »

Le ton plein d'humour et le cynisme sous-jacent révèlent l'observation aiguisée de ce monde marchand. Mais l'artiste sait que cette distance critique qu'il instaure est aussi celle qui lui permettra de garder son auto-

- 12. À souligner, Musée d'art moderne, Département des Aigles apparaît toujours en italique, mentionné comme un titre, on comprend qu'il occupe au-delà de sa fonction muséale fictive ou virtuelle, avant tout le statut d'œuvre dès lors qu'il est cité, inventorié ou répertorié.

13. Cette déclaration célèbre de Marcel Broodthaers a été publiée dans de nombreux ouvrages qui lui ont été consacrés de son vivant et surtout après sa mort. Nous l'avons reprise dans le catalogue d'exposition de la Tate Gallery, Londres, 1980, p. 12. La citation partielle de la déclaration est aussi reprise sur la couverture du catalogue d'exposition de la rétrospective que la Galerie nationale du Jeu de paume lui consacre en 1992. 
nomie, même s'il s'agit de confirmer ses besoins financiers et de souligner la réalité économique de l'art.

Dans le contexte des événements de mai 1968, Broodthaers participe à l'occupation du Palais des beaux-arts de Bruxelles, avec d'autres artistes, intellectuels, étudiants et activistes qui souhaitent se montrer solidaires des nombreuses manifestations politiques dans les pays européens mais, dans le même temps, s'inscrire dans un lieu symbolique afin de contester la manière dont les institutions officielles belges exercent un contrôle tant artistique que financier sur la culture de leur pays. Quelques mois plus tard, le 27 septembre 1968, Marcel Broodthaers inaugure de façon officielle le premier département de son Musée d'art moderne, Département des Aigles, dans son appartement bruxellois de la rue de la Pépinière qui lui sert aussi d'atelier. Nommé « Section XIX siècle », il est composé d'une trentaine de caisses de transport d'art vides avec les instructions habituelles inscrites au pochoir («Fragile », « À manier avec précaution », etc.), de trente cartes postales montrant des reproductions de peintres français du XIX ${ }^{\mathrm{e}}$ siècle (Courbet, Corot, Delacroix, Ingres, Meissonier, Puvis de Chavannes), d'une échelle appuyée sur un mur. Des numéros sur chacune des portes de chambre les transforment en galeries. Les mots « Musée/Museum » inscrits sur les fenêtres sont lisibles de l'extérieur. Pendant la soirée inaugurale, une projection de diapositives présentant les gravures de Grandville est organisée. Pour convier les invités à cette soirée (une soixantaine de personnalités du monde de l'art était présente, Johannes Cladders, directeur du musée d'État de Mönchengladbach y prononce un discours), Marcel Broodthaers envoie un courrier « officiel » avec comme en-tête « Cabinet des Ministres de la Culture » et comme signature « Pour l'un des Ministres, Marcel Broodthaers ». La lettre dit : « Nous avons le plaisir d'annoncer aux consommateurs et aux curieux l'ouverture du "Département des Aigles" du Musée d'art moderne. Les travaux sont en cours de réalisation ; leur achèvement déterminera leur date, moment où nous espérons que la poésie et les arts plastiques pourront marcher ensemble. Nous espérons que notre formule de "désintéressement plus admiration" vous séduira ${ }^{14}$. » Le Musée reste ouvert pendant une année rue de la Pépinière, avant de se rendre à Anvers pour une semaine (le 27 septembre 1969), où il s'installe dans une galerie d'art alternative dirigée par Kaspar König, dont le nom correspond au numéro de téléphone du lieu, A 3790 89. Cette nouvelle section est intitulée «Section XVII ${ }^{\mathrm{e}}$ siècle ». Comme pour la première section, des caisses de transport vides sont arrangées dans l'espace et des cartes postales de toiles de Rubens sont accrochées sur les murs. L'inscription « Département des Aigles » est placée avec de larges lettres sur le mur côté jardin. Cinq mois plus tard, Broodthaers emprunte à la collection du Kunstmuseum de Düsseldorf huit peintures XIX ${ }^{e}$ de l'école allemande locale pour les installer à la Kunsthalle de Düsseldorf, dans le cadre d'une exposition intitulée Between 4 et organisée par le conservateur adjoint de l'institution Jürgen Harten. Sur le mur opposé, sont accrochées les cartes postales

14. Marcel Broodthaers, cité par Douglas Crimp, "This Is Not A Museum », in On the Museum's Ruins (avec des photographies de Louise Lawler), Cambridge MA, The MIT Press, 1993, p. 205. 
de la première section ainsi que des reproductions montrant l'installation dans l'appartement de Bruxelles. Broodthaers joue ici sur la juxtaposition entre les œuvres réelles (dans le sens de originales et authentiques) et les images reproduites, qui renvoient à une forme de fiction de l'art (cartes postales...). La remise en cause de l'original propre aux pratiques de beaucoup d'artistes des années 1960 et 1970 rejoint les théories développées sur la notion d'auteur. Ce déplacement de l'original vers la copie en passant par le médium photographique rappelle le Musée imaginaire, projet monumental d'André Malraux. C'est cet exemple que Douglas Crimp reprend dans On the Museum's Ruins et il n'est pas anodin de souligner la traduction anglaise du Musée imaginaire qui devient «Museum without Walls», le « Musée sans murs », qui appuie le processus que développe précisément Broodthaers avec son Musée d'art moderne. "Toute œuvre d'art qui peut être photographiée a sa place dans le "super" musée [supermuseum] de Malraux », précise Crimp. Il fait aussi référence à la méthode de l'historien d'art qui, grâce au diaporama projeté pendant ses cours, possède cette

Fig. 11 : Marcel Broodthaers, Musée d'art moderne, Département des Aigles,

(a) "Section xIx ", Bruxelles, sept. 1968, Anvers, 1969,

(b) avec Jûrgen Harten, "Section xix ${ }^{\mathrm{e}}$ bis ", Städtische Kunsthalle, Düsseldorf, 1970,

(c) couverture du catalogue, "Section financière ", Foire de Cologne, 1971.

(a) 
liberté de juxtaposer des images d'œuvres d'artistes et d'époques différentes, rendant par là même possible des raccourcis historiques permettant de créer des sens esthétiques et des parallèles formels renouvelés. Pour Crimp, le Musée imaginaire se nourrit de ces projections. Dans le cadre du projet de Broodthaers, la confrontation entre les originaux et les copies renvoie aussi à une réalité économique : qui peut acheter une toile de maître et qui achète une carte postale ? Mais la fonction de la copie rejoint aussi celles du document et de l'archive.

L'étape suivante du Musée d'art moderne est une halte d'une journée sur une plage belge de la mer du Nord. Marcel Broodthaers et son ami collectionneur Herman Daled font un château de sable en forme de musée en portant des chapeaux de toile sur lequel le mot «musée » est écrit. Ils placent des signes autour du musée de sable où on peut lire : « Toucher les objets est formellement interdit. » Au-delà de l'aspect ludique, la gravité du sens qui émerge dans ce travail de Broodthaers renvoie précisément à cette institution muséale qui a fondé comme un mausolée un certain nombre des principes immuables du rapport que l'on entretient avec les œuvres d'art. La critique de Broodthaers vis-à-vis du musée laisse ici entendre qu'un coup de vent détruira l'architecture de sable mais pourra aussi laisser sur la paille les artistes qui en dépendent. C'est en tenant compte de cette réalité que, avec ironie, il ouvre une nouvelle section de son musée, la "Section financière », dans le cadre de la Foire de Cologne en 1971. On peut lire sur un tract reprenant la forme d'une jaquette de livre : «Musée d'art moderne à vendre pour cause de faillite. » Il renverse dès lors dans le contexte d'une foire, symbole économique du marché par excellence, le statut de son musée fictif en un produit pour le marché de l'art. C'est dans le cadre de cette "Section financière »

(b) 
qu'il introduit également la vente de multiples, une série illimitée de lingots d'or estampillés de la signature de l'artiste.

En janvier 1971, Broodthaers propose la «Section cinéma » dans un sous-sol loué à Düsseldorf où sont notamment projetés ses propres films et ceux de Chaplin. Est également projeté sur une carte du monde un film de l'artiste réalisé en 1969 et intitulé Le Musée et la Discussion. D’autres manifestations ponctuelles y sont organisées pendant toute l'année.

L'une des sections centrales du projet de Broodthaers est celui qu'il inaugure à la Kunsthalle de Düsseldorf en 1972. La « Section des figures » propose trois cents objets et images représentant des aigles choisis sur une période qui va de l'époque Oligocène (des milliards d'années avant notre ère) jusqu'au présent. Ces œuvres sont empruntées auprès de nombreux musées et de collectionneurs privés. L'une des pièces propose une installation encyclopédique qui rappelle les cabinets de curiosités. Toutes les œuvres sont accompagnées d'un cartel qui indique : «Ceci n'est pas une œuvre d'art. » Pour Broodthaers, cette phrase est une référence directe au fameux titre du tableau Ceci n'est pas une pipe que René Magritte a peint en 1926. Contredisant le sens même de la représentation en créant de façon elliptique un rapport à la négation de ce qui est donné à voir, cette phrase renverse la tautologie que des artistes conceptuels comme Joseph Kosuth ont pu poser, en soulignant le fait que l'artiste s'octroie la liberté de définir lui-même le statut des objets présentés. La dernière étape de son Musée se tient en 1972 dans le cadre de la Documenta, fameuse manifestation internationale d'art contemporain qui se tient tous les cinq ans dans la ville allemande de Kassel. Harald Szeemann, commissaire de l'exposition pivot Quand les Attitudes deviennent Formes (1969), en est le directeur artistique.

Le Musée d'art moderne, Département des Aigles de Broodthaers reste le modèle unique et absolu d'un projet qui prend comme objet d'étude toutes les pratiques muséales dans leur ensemble, du transport à la conservation, de l'exposition à la collection, de la taxinomie à l'archive. Cette recherche rappelle, comme le souligne Douglas Crimp lorsqu'il fait référence à la «Section des figures », la méthode foucaldienne définie notamment dans L'Archéologie du savoir en 1969.

«Le but de l'archéologie de Foucault, écrit Crimp, est de montrer que le site qui permet de juxtaposer des entités hétérogènes est celui du discours et que ces formations discursives peuvent subir des mutations historiques d'une telle échelle qu'elles peuvent devenir complètement incompatibles les unes avec les autres ${ }^{15}$. »

L'exigence avec laquelle Marcel Broodthaers construit sa démarche artistique et critique fait de lui une référence pour les artistes des générations suivantes formés avec une rigueur intellectuelle qui jouxte une parfaite connaissance historique, elle-même nourrie par une maîtrise formelle des 
$œ u v r e s$ et de leur exposition. Cette confrontation visuelle est celle qui permet de faire jaillir du sens lorsque les limites du musée sont révélées.

C'est suivant une méthode similaire que Fred Wilson (né à New York en 1954) procède depuis la fin des années 1980. Concevant sa pratique artistique comme une critique de l'institution, il devient pour les directeurs et les commissaires qui prennent le risque de l'inviter dans leur musée, un élément extérieur clé leur permettant de regarder autrement leurs collections et leurs archives. La façon dont il pense le dispositif muséal en s'appuyant sur une lecture transversale des disciplines comme l'histoire de l'art, l'anthropologie, l'ethnologie ouvre pour le public qui découvre ses expositions un agencement inédit révélant les absences, renouant avec des héritages culturels, interrogeant la place de l'œuvre en la renvoyant à son contexte de production. Analysant les différents procédés de représentation au sein d'un musée, il construit une réflexion qui se fonde sur la manière dont l'institution impose un regard sur les œuvres ou les objets qu'elle présente.

Dans un essai qu'elle consacre à l'artiste, Mining the Museum, Artists look at Museums, Museums look at Themselves, Lisa G. Corrin énonce un point de vue particulièrement frontal sur le musée : «Parler du dispositif idéologique qui sous-tend les pratiques muséographiques, c'est parler de la relation entre le pouvoir, la représentation et l'identité culturelle, parler de la façon dont l'histoire est construite et médiatisée, à qui appartient cette histoire, ce qu'elle révèle, ce qu'elle tait. Derrière leurs halls souvent caverneux de reliques culturelles, les musées sont des lieux où les sacro-saints systèmes de croyance sont confirmés sur la base d'une hiérarchie qui évalue une culture par rapport à une autre ${ }^{16}$. »

En 1987, Wilson organise une exposition dans le Bronx (où il est né), qu'il intitule Rooms with a View: The Struggle Between Culture, Content and Context (Chambres avec vue : lutte entre culture, contenu, contexte). Chacun des trois espaces composant l'installation simule un espace muséal distinct : le premier fait référence au musée ethnographique, le second à la période victorienne, le dernier à l'espace « blanc » conventionnel - le « cube blanc ». Les œuvres de près de trente artistes occupent les trois espaces ; elles sont regroupées selon des distinctions similaires aux trois pièces en question. Ainsi le musée ethnographique présente des objets d'art en précisant vaguement leurs matériaux, mais sans indiquer leur auteur; le «musée victorien » montre les objets comme des antiquités, posées sur de précieux piédestaux ; enfin, le « cube blanc » accueille des œuvres plutôt tranchantes suggérant l'art contemporain. Cette expérience oriente Wilson vers un processus créatif qui choisit comme sujet le musée lui-même, avec ses paramètres culturels, sociaux et politiques. L'objectif de l'artiste est de démontrer que la construction du musée occidental n'est effective que du seul point de vue occidental mais qu'il ne prend pas suffisamment en

16. Lisa G. Corrin, " Mining the Museum: Artists look at Museums, Museums look at themselves », dans le catalogue de l'exposition Fred Wilson: Mining the Museum, Maryland, Maryland Historical Society, 1992, p. 1. 
Fig. 12 : Fred Wilson, Untitled (Atlas), 1992.

considération les pratiques ou créations de la périphérie, souvent invisibles pour le mainstream.

Devenu sujet, et non plus contenant, le musée tourné en dérision par Fred Wilson devient dans le même temps un outil de travail expérimental : «Tout l'environnement du musée est devenu ma palette, mon vocabulaire. J'observe tout et j'essaie de tout distiller, afin de réutiliser, de réinventer, le sens qui s'en dégage ${ }^{17}$. » Parasitant l'espace muséal, ses projets consistent à intégrer au sein du musée des objets qui, achetés à des vendeurs de rue par exemple, ne possèdent pas la valeur d'une œuvre d'art et qui, par là, tendent à déstabiliser l'équilibre des œuvres habituellement présentées. En 19901991, lors de son projet The Other Museum, à Washington, il présente des masques africains dont les « yeux » sont recouverts de drapeaux français et anglais sous forme de bandeaux, suggérant de façon explicite le pouvoir colonial. Sous d'autres masques, on peut lire un cartel qui indique « volé à la tribu Zonge ». Il souligne la présentation des masques par des éclairages de couleur et indique par là son souci de montrer comment les musées " anesthésient l'importance historique de ces objets en vue de recouvrir l'histoire coloniale ${ }^{18} »$.

Pour Maurice Berger, la liberté que lui accordent les directeurs de musée le rapproche de la position de l'archéologue qui déterre des objets enfouis et ignorés afin de révéler des histoires cachées ${ }^{19}$.

19. Voir Maurice Berger, "Viewing the Invisible, Fred Wilson's Allegories of Absence and Loss ", in Fred Wilson, Objects and Installations 1979-2000, Baltimore, Center for Art and Visual Culture, University of Maryland, 2001, p. 8-20. 
Fig. 13 : Fred Wilson, vue de l'exposition Primitivism: High and Low, Metro Pictures Gallery, New York, 1991.

À gauche : Guarded View (4 mannequins noirs sans tête portant les uniformes des gardiens de quatre musées new-yorkais) ; à droite : Picasso/Whose Rules?, reproduction échelle 1 des Demoiselles d'Avignon de Picasso (1907), avec un masque en bois Kifwebe.

En 1992, l'artiste s'engage, pour une durée de un an, dans un projet qui consiste à créer un modèle alternatif remettant en cause l'espace de l'art institutionnel ainsi que la représentation de type muséal. Pour la première fois depuis le début de sa recherche sur ce thème, Wilson a la possibilité de travailler au sein d'un musée historique traditionnel, la Société historique du Maryland. Il a de surcroît un statut différent : outre celui d'artiste, il occupe la position d'un directeur artistique, avec le contrôle du projet d'exposition dans son intégralité. Intitulée Mining the Museum (Miner le musée), l'exposition propose une réflexion générale sur l'appareil idéologique du musée et son oubli fréquent de l'histoire des Noirs américains. Il commence une étude approfondie des œuvres et des objets présents dans la collection de la Maryland Historical Society, ainsi qu'une recherche précise au sein des archives de l'institution. Cette dernière lui permet de remonter jusqu'à la période de l'esclavage au XIX ${ }^{\mathrm{e}}$ siècle et de retenir un certain nombre de documents relatifs à son histoire. Il découvre des papiers administratifs ayant appartenu aux esclavagistes, des contrats de vente d'esclaves, des lettres : «J'étais vraiment stupéfait par la richesse qui ressortait de toutes ces transactions. Voir la juxtaposition de toutes ces choses était vraiment pénible. [...] Avant que je ne m’engage dans ce projet, je pensais plus au colonialisme et à l'Afrique qu'à l'Amérique et à l'esclavage des Noirs. Je n'ai pas choisi de travailler sur l'esclavage, j'ai découvert des indices ${ }^{20}$. »

En déterminant les éléments qu'il va ou non intégrer à son installation (celle-ci occupe entièrement le troisième étage du musée et comprend plus d'une centaine de pièces toutes relatives à l'histoire noire américaine), il questionne la manière dont les musées américains représentent - ou plutôt ne représentent pas - les spécificités de la culture africaine-américaine et la façon dont l'histoire des Noirs a été largement occultée par le mainstream.

20. Leslie King-Hammond, "A Conversation with Fred Wilson », Fred Wilson: Mining the Museum, op. cit., p. 33. 
Tout en se situant au croisement d'une critique institutionnelle et d'une politique identitaire multiculturelle, le projet de Fred Wilson a été vu par un nombre important de visiteurs et a reçu un accueil très positif de la part du monde de l'art autant que de la presse quotidienne locale.

À la suite de cette expérience, Wilson est invité l'année suivante par le Art Museum de Seattle pour y concevoir un projet similaire. Différence remarquable, le premier musée était un musée d'histoire et celui-ci est un musée d'art. L'artiste explique cette différence en indiquant notamment que dans le Maryland, il a insisté sur la possibilité de faire une histoire de la culture noire à partir des objets de la collection comme il aurait été possible de construire avec d'autres œuvres du musée une histoire des femmes ou une histoire de l'immigration. Son choix de l'histoire africaine-américaine et des Indiens d'Amérique dépend évidemment de critères subjectifs qu'il revendique pleinement. C'est aussi pourquoi il ne s'agit pas pour lui de penser son projet comme une histoire noire exhaustive. Le projet à Seattle, lui, oriente son travail vers une autre direction. Quittant le domaine de la documentation historique ou de l'ethnographie, il s'inscrit dans une institution artistique très récente (une année d'existence) alors que la Société historique du Maryland remontait au milieu du XIX ${ }^{e}$ siècle. Le processus artistique s'en trouve bien évidemment modifié.

La collection du musée de Seattle réunit des œuvres et pièces d'art du monde entier. Fred Wilson souligne de façon ironique que les conservateurs de ces musées peuvent s'enorgueillir de posséder des collections «multiculturelles » mais que, pour lui, « c'est aussi multiculturel que l'Empire britannique : toutes les cultures sont là mais qui décide de ce qu'elles ont à dire ? Qu'est-ce qui doit se trouver à côté de telle ou telle chose ? À quel degré d'importance ${ }^{21} »$ ? C'est ainsi qu'à partir du plan du musée et de la disposition des collections en son sein, en observant, par exemple, à quel étage se trouve l'Antiquité, avec l'Égypte, la Grèce, Rome, puis l'époque médiévale et la Renaissance en Europe et ce, jusqu'à l'art américain du XXe siècle, l'artiste va réaliser une nouvelle organisation des accrochages et des vitrines, en juxtaposant les œuvres selon sa propre logique artistique.

Le déplacement entre Maryland et Seattle permet aussi à l'artiste d'aller au-delà d'un questionnement propre à son identité, en privilégiant, sans renoncer à cette dernière, sa qualité d'artiste avant celle d'homme noir. La problématique de Fred Wilson se situe dès lors dans la continuité des analyses sur les artistes qui investissent leur propre identité ethnique ou raciale, et développent des "stratégies » esthétiques et politiques pour construire une critique effective d'une société américaine au sein de laquelle les Africains-Américains ont peu d'espace de mobilité.

Deux exemples représentatifs ont été choisis comme étude de cas mais les artistes qui intègrent la problématique du musée dans le travail de création sont loin d'être isolés. Depuis les années 1960, les expériences 
plus alternatives ont laissé la place à des situations institutionnalisées où les artistes invités à contredire les règles muséales travaillent en corrélation avec les commissaires d'exposition et les directeurs de musée. Ces derniers suivent de plus en plus fréquemment des questionnements qui s'approprient les méthodes critiques de certains artistes conceptuels. Comme l'énonce Rudi Fuchs, en charge du Van Abbemuseum d'Eindhoven en 1977, et ayant accueilli les artistes les plus exigeants des années 1970 : «Le directeur prend le musée comme il est : il le voit (du moins c'est ma

position) comme un instrument qui peut s'étirer à son maximum afin de satisfaire la plus grande variété de productions artistiques et d'attitudes qui composent chaque année la programmation ${ }^{22}$.»

Dans le contexte d'une société contemporaine globalisée qui accueille aujourd'hui des pratiques artistiques mondiales au sein d'institutions elles-mêmes créées chaque jour sur les cinq continents, la question du musée contemporain se lit, au-delà de son inscription première dans le monde occidental, à la lumière d'une cartographie qui permet la fondation de nouveaux socles de savoir. Les artistes, les directeurs de musée, les critiques et historiens de l'art ont la responsabilité de retracer la généalogie d'une histoire qui se construit dans une forme rhizomique d'échanges culturels. Plus que jamais, la fonction de l'archéologie définie par Foucault est utile pour comprendre le paradigme idéologique du musée. Les ruptures historiques, les transformations esthétiques, les propositions théoriques, les strates du savoir nous permettent de confirmer que la pensée critique accompagne la création artistique là où elle se trouve. Ici et maintenant.

historienne de l'art contemporain, maître de conférences à l'université Rennes-2

22. Rudi Fuchs, Michael Asher Exhibitions in Europe 1972-1977, Eindhover, Van Abbemuseum, 1980, p. 34. 